\title{
Free-breathing 3D whole-heart coronary mra using respiratory motion-resolved sparse reconstruction
}

\author{
Davide Piccini ${ }^{1,2^{*}}$, Li Feng ${ }^{3}$, Gabriele Bonanno ${ }^{1,4}$, Simone Coppo ${ }^{2,4}$, Jérôme Yerly ${ }^{2,4}$, Ruth P Lim ${ }^{5}$, Juerg Schwitter ${ }^{6}$, \\ Daniel K Sodickson ${ }^{3}$, Ricardo Otazo ${ }^{3}$, Matthias Stuber ${ }^{2,4}$ \\ From 19th Annual SCMR Scientific Sessions \\ Los Angeles, CA, USA. 27-30 January 2016
}

\section{Background}

Navigator gating is commonly used to minimize respiratory motion in free-breathing whole-heart coronary MRA [1]. However, lengthy and unpredictable acquisition times remain a drawback. Respiratory self-navigation (SN) [2-3], conversely, enables $100 \%$ scan efficiency, but performs motion correction over a broad range of respiratory displacements, which can result in image artifacts. Here, we propose an alternative respiratory motion-resolved approach based on 3D radial phyllotaxis sampling, respiratory motion sorting and sparse reconstruction.

\section{Methods}

Examinations in $\mathrm{N}=11$ healthy volunteers (9 male, age: $29 \pm 4$ y) were performed on a $1.5 \mathrm{~T}$ clinical MRI scanner (MAGNETOM Aera, Siemens Healthcare) with a prototype 3D radial phyllotaxis bSSFP sequence [4]: TR/TE 3.1/ $1.56 \mathrm{~ms}$, FOV $(220 \mathrm{~mm})^{3}$, matrix $192^{3}$, voxel $(1.15 \mathrm{~mm})^{3}$, $\mathrm{RF}$ angle $115^{\circ}$, and receiver $\mathrm{BW} 898 \mathrm{~Hz} / \mathrm{Px}$. Using a respiratory signal directly extracted from the modulations of the k-space center amplitude within the radial imaging data [5], signal-readouts were grouped according to the respiratory state at which they were acquired (Fig. 1). The resulting series of undersampled respiratory states were reconstructed using an eXtra-Dimensional Golden-angle RAdial Sparse Parallel imaging (XD-GRASP) [6] algorithm, which exploits sparsity along the newly created respiratory dimension. Datasets for 4 respiratory states were reconstructed. Image quality of the end-expiratory phase was compared to $1 \mathrm{D}$ respiratory self-navigation in terms of vessel sharpness (VS) [7], visible length (VL) and diagnostic quality on a scale from 0 (non-visible) to 2 (diagnostic).

\section{Results}

Respiratory-resolved XD-GRASP reconstruction effectively suppresses respiratory motion artifacts (Fig. 1). Average VS and VL were always superior for the respiratory-resolved datasets, reaching statistical significance $(\mathrm{p}$ $<0.05$ ) for the left main (LM), for the proximal and mid left anterior descending artery (LAD) (e.g. VS of mid LAD $40.8 \pm 9.1 \%$ vs $34.9 \pm 10.2 \%$ ) and for the mid right coronary artery (RCA). Visualized length of LM+LAD was significantly increased as well. A total of $41 / 88$ coronary segments were graded as diagnostic for 1D SN, while this ratio increased to $61 / 88$ for the XD-GRASP reconstruction (Tab.1). The XD-GRASP reconstruction reached $100 \%$ diagnostic quality for LM, proximal-LAD, and proximal-RCA.

\section{Conclusions}

Instead of discarding data or enforcing motion models for motion correction, XD-GRASP makes constructive use of all respiratory phases to improve image quality, and achieves superior quality compared to $1 \mathrm{D}$ respiratory SN without the need for breath-holding, navigators, or complex 3D respiratory motion correction schemes. The phyllotaxis trajectory and XD-GRASP reconstruction provide a synergistic combination that may lead routine coronary MRA closer to clinical practice. 


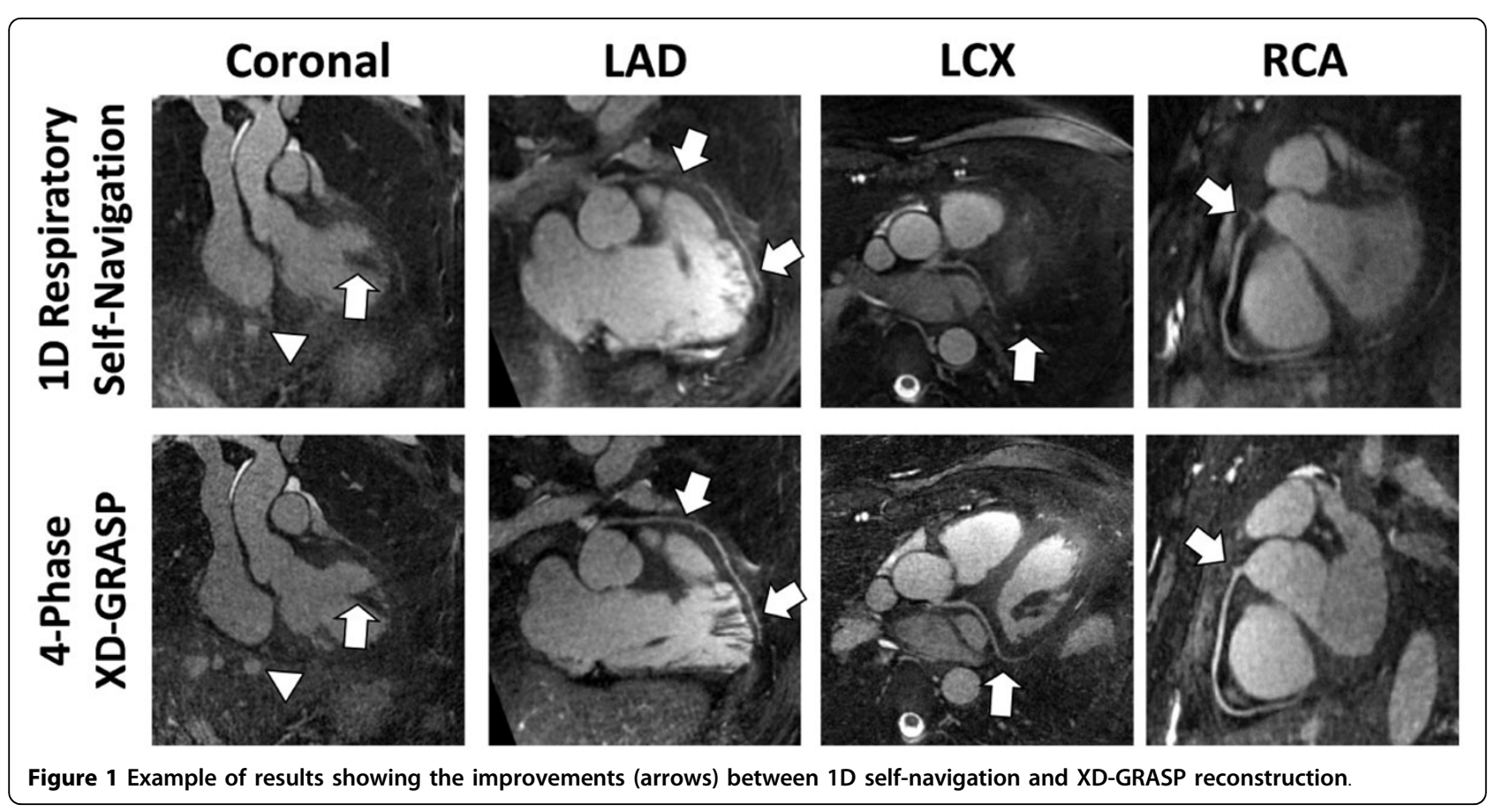

Table 1 Diagnostic quality grading of all coronary segments

\begin{tabular}{ccc}
\hline Coronary Segment & 1D Respiratory Self-Navigation & 4-Phase X-D GRASP (End-exp) \\
\hline Left Main & $1.8 \pm 0.4$ & $2.0 \pm 0.0^{*}$ \\
LAD Prox. & $1.6 \pm 0.5$ & $2.0 \pm 0.0^{*}$ \\
LAD Mid & $1.3 \pm 0.6$ & $1.4 \pm 0.5$ \\
LAD Dist. & $0.9 \pm 0.5$ & $1.3 \pm 0.5$ \\
LCX Prox. & $1.4 \pm 0.7$ & $1.4 \pm 0.7$ \\
RCA Prox. & $1.8 \pm 0.4$ & $2.0 \pm 0.0$ \\
RCA Mid & $1.3 \pm 0.5$ & $1.7 \pm 0.5$ \\
RCA Dist. & $1.4 \pm 0.7$ & $1.7 \pm 0.5$ \\
\hline Total Diagnostic Segments & $41 / 88(47 \%)$ & $61 / 88(70 \%)$
\end{tabular}

All values are expressed as mean \pm one standard deviation

* Indicates statistical significance compared to 1D Respiratory Self-Navigation.

Diagnostic Grading: $0=$ non-visible, $1=$ visible but non diagnostic and $2=$ visible and diagnostic

\section{Authors' details}

${ }^{1}$ Advanced Clinical Imaging Technology, Siemens Healthcare, Lausanne, Switzerland. ${ }^{2}$ Department of Radiology, University Hospital (CHUV) and University of Lausanne (UNIL), Lausanne, Switzerland. ${ }^{3}$ Department of Radiology, Center for Advanced Imaging Innovation and Researc (CAI2R) and Bernard and Irene Schwartz Center for Biomedical Imaging, New York, NY, USA. ${ }^{4}$ Center for Biomedical Imaging (CIBM), Lausanne, Switzerland.

${ }^{5}$ Department of Radiology, Austin Health and The University of Melbourne, Melbourne, VIC, Australia. 'Division of Cardiology and Cardiac MR Center, University Hospital of Lausanne (CHUV), Lausanne, Switzerland.

Published: 27 January 2016

Submit your next manuscript to BioMed Central and take full advantage of:

- Convenient online submission

- Thorough peer review

- No space constraints or color figure charges

- Immediate publication on acceptance

- Inclusion in PubMed, CAS, Scopus and Google Scholar

- Research which is freely available for redistribution 